\title{
Las diásporas en la arena internacional: el caso de la diáspora india
}

\section{Lía Rodríguez de la Vega*}

\section{RESUMEN}

Se estima que, en la actualidad, 214 millones de personas viven fuera de su país de origen (oIM, 2010). El fenómeno migratorio es traducido en este estudio como diáspora y ha sido vinculado a los distintos actores no estatales que ejercen su influencia a nivel local y global. Sheffer (1986) señala que las redes creadas por diásporas étnicas forman parte de una relación triádica que comprende al país de origen, la diáspora y los países de residencia, y apunta que esa relación deviene en un aspecto integral y permanente del flujo de política de ambos niveles.

Este trabajo aborda el caso del segmento de la diáspora india asentada en Estados Unidos y su influencia en la política exterior de la India, a través de distintas acciones y procesos políticos. Se consideran para ello diversos autores que han abordado el tema y el reporte hecho por el Comité de Alto Nivel sobre la Diáspora India. Se concluye que distintas acciones de este segmento de la diáspora evidencian la ligazón de la esfera política internacional con la doméstica (de India) y su promoción de los intereses de la India como grupo de presión, aunque el alcance de su accionar resulta aún limitado, debido al pequeño volumen de sus votantes, por lo que precisa profundizar su accionar en el congreso del país de residencia, y, a su vez, debe extenderse a otras acciones, destacándose también en ese panorama el accionar de individuos y agentes económicos indios.

Palabras clave: Diáspora india, identidad, articulación local/global, "poder blando"/ diplomacia.

* Doctorado en Relaciones Internacionales. Responsable del Área de Asia y África de unicom, Facultad de Ciencias Sociales de la Universidad Nacional de Lomas de Zamora; investigadora de la Universidad de Palermo. Buenos Aires (Argentina). liadelavega@hotmail.com

Recibido: 4 de abril de 2015 / Modificado: 3 de junio de 2015 / Aceptado: 3 de junio de 2015

Para citar este artículo

Rodríguez de la Vega, L. (2015). Las diásporas en la arena internacional: el caso de la diáspora india. OASIS, 22, 23-47. DOI: http://dx.doi.org/10.18601/16577558.n22.03 


\section{Diasporas in the International Arena:The Case of the Indian Diaspora}

\section{ABSTRACT}

The oIm (2010) estimates that at present, 214 million people live outside of their country of origin. The migratory phenomenon is seen in this study as that of diaspora and has been linked to the different non-state actors who exercise their influence at the local and global levels. Sheffer (1986) notes that the networks created by ethnic diasporas are a part of a relationship between the country of origin, the diaspora and the countries of residence, pointing out that this relationship occurs as an integral and permanent aspect of the flow of politics at both levels.

This work focuses on the case of the segment of the Indian diaspora placed in the United States and its influence on the foreign policy of India, across different actions and political processes. To do that, we consider different authors who have studied the topic and the report on the Indian diaspora done by the High Level Committee on Indian Diaspora. Conclusions indicate that different actions of this segment of the diaspora demonstrate the attachment of the international political sphere with the domestic one (of India), and its promotion of the interests of India, as a lobbying group. However, the scope of its actions turns out to be limited due to the small volume of its voters. Due to this, it needs a deeper approach in the American congress, while at the same time expanding toward other actions. The actions of individuals and Indian economic agents also stand out in this panorama.

Keywords: Indian diaspora, identity, Local-global articulation, soft power/diplomacy.

\section{INTRODUCCIÓN}

En la actualidad, de acuerdo con lo sostenido por la Organización Internacional para las Migraciones (OIM) (2010), se estima que 214 millones las personas viven fuera de su país de origen, representando el 3\% de la población mundial. El fenómeno migratorio supera el plano nacional, pudiendo influir en las relaciones interestatales y de distintos actores no estatales, y constituirse en instrumento de la política exterior, con efectos al interior y exterior tanto de los países emisores como de los receptores.

Las comunidades emigradas, bajo la denominación de diáspora, aparecen entre los actores no estatales, entidades no soberanas que ejercen una influencia significativa tanto a escala nacional como global (Gupta, s. f.). Al respecto, Sheffer (1986) señala que las redes creadas por diásporas étnicas poseen características particulares dado que forman parte de una relación triádica que comprende al país de origen, la diáspora misma y los países de residencia, apuntando que esa relación deviene en un aspecto integral y hasta permanente del flujo de política de ambos niveles. Esta relación evidencia dinámicas que articulan lo global y lo local, y abarcan distintas temáticas, especialmente asuntos relativos a lo identitario y los recursos y niveles de influencia. Agrega que las diásporas étnicas surgen por migración 
voluntaria o forzada y se asientan en uno o más países, en los que permanecen como grupos minoritarios, preservando su identidad étnica y su solidaridad comunal, siendo este último elemento el que permite mantener y promocionar el contacto entre elementos activistas de estas diásporas que a su vez poseen significado político, económico, cultural, etc., para las diásporas mismas, sus países de residencia y el país de origen.

De ese modo, la dinámica transnacional de las diásporas se relaciona con el "nacionalismo de larga distancia”, enunciado por Anderson (1993), el "nacionalismo diaspórico" enunciado por Gellner (1988), etc., atendiendo a que las migraciones modificaron lo que anteriormente parecía ser una coincidencia natural entre un sentimiento nacional y la residencia en la tierra de origen.

La consideración de los grupos diaspóricos ha derivado en un alcance interdisciplinario en el campo de las ciencias sociales, con conexiones teóricas entre las distintas disciplinas, que traen al centro de la cuestión distintas nociones asociadas al fenómeno abordado, tales como cultura, identidad, comunidad, vínculos primordiales y otros vínculos (Geertz, 1963), autoimagen (Zolberg y Woon, 1999), gestión de la diversidad cultural (Kymlicka, 1996), seguridad, etc.

Este trabajo se propone abordar el caso del segmento de la diáspora india asentada en Estados Unidos, y su influencia en la política exterior de su país de origen, a través de distintas acciones y procesos políticos. Para ello, se consideran diversos autores que han abordado esta temática y otras relacionadas, y el reporte que sobre el tema hizo el Comité de Alto Nivel sobre la Diáspora India comisionado para ello (MEA, 2002).

\section{BREVE ESTADO DE LA CUESTIÓN}

Al considerar la numerosa producción existente en las ciencias sociales sobre el fenómeno migratorio, Mitchell (2012) sostiene que el advenimiento de un nuevo orden trajo consigo el reconocimiento, por parte de los académicos, de una gran variedad de nuevos actores (sobre todo no estatales), previamente ignorados en política internacional. En tal sentido, en lo que respecta a la temática de la migración / diáspora en las relaciones internacionales, apunta que, ignorada por largo tiempo, recapturó la atención tras el episodio de septiembre de 2001, resultando de ello trabajos principalmente enfocados en la seguridad (asociados al control fronterizo, terrorismo, refugiados, solicitantes de asilo, el nexo con conflictos ambientales y el rol específico de las diásporas en las relaciones internacionales). Agrega que, en la actualidad, la temática migratoria es considerada como de "alta política" en la nueva agenda política, y que si bien se han registrado avances en su estudio en el marco de las relaciones internacionales, la comprensión de las implicancias políticas de las migraciones es aún limitada.

Ya Weiner (1985) plantea que el modo como el Estado modela los movimientos de población, cuándo estos llevan a situaciones de conflicto o de cooperación y qué hacen los gobiernos en sus políticas domésticas para influenciar los flujos migratorios son preguntas que han recibido poca atención. Hollifield (2008) da una respuesta a tal situación, comentando que el periodo que se extiende entre 
1945 y 1990 estuvo dominado por la Guerra Fría, y en ese periodo los teóricos tendían a dividir la política en alta y baja política, y como la migración no afectaba de manera directa el balance de poder, la disputa este-oeste o la naturaleza del sistema internacional -con excepción de los refugiados-, no logró llamar la atención de los investigadores. Miller (1998), por su parte, apunta que la proliferación de actividad diplomática en relación con cuestiones migratorias, y el avance de la integración socioeconómica, entre otras razones, contribuyeron a visibilizar los nexos de la temática de la migración internacional con el de la seguridad. Distintos estudios dan cuenta de ello: Matthews (1993), Brochmann y Hammar (1999), Rudholp (2003), Cornelius, Takeyuki, Philip y Hollifield (1992, 2004, 2012), Adamson (2006), Dannreuther (2007), entre otros.

El abordaje de las migraciones en el marco de la seguridad, trata también la relación existente (o no) entre conflictos y medioambiente, siendo algunos ejemplos, los trabajos de Deudney (1990), Gleditsch (1998), Homer-Dixon (1999), Reuveny (2007), entre otros.

En lo relativo a la migración forzada (asociada a trata y tráfico de personas, refugio, desplazamiento y asilo) y su relación con la política internacional, Mitchel (2012) apunta que hay pocos trabajos que consideren la temática, pudiendo mencionarse entre ellos a Frechette (2004), Betts (2009, 2014), Kauffman (2015), etc.

En lo que hace a otras diversas cuestiones asociadas a los migrantes, González Gutiérrez
(2006) reúne autores de diversos países, que abordan temáticas tales como el marco institucional para el diseño y la implementación de políticas públicas dirigidas a las comunidades residentes en el exterior; los derechos políticos para la diáspora en su comunidad de origen (y en algunos casos, también en su comunidad de residencia); las políticas educativas y de salud, y considera las implicancias que para la identidad del país de origen tiene el saberse tal lugar de poblaciones diaspóricas. Xiang (2005), a partir del estudio de la manera como China ha formado parte históricamente del sistema internacional moderno y de su percepción de la migración en conexión con su estrategia diplomática, aborda la valoración de la relación entre migración transnacional y las relaciones internacionales. Mientras Zeraoui (2006) se ocupa de la problemática global de las migraciones a nivel mundial y su relevancia en la coyuntura actual, para poder centrarse luego en el caso de África, Postelnicu (2010) estudia la idea de los bucles de retroalimentación entre los migrantes que retornan a su país de origen, o entre los repatriados y el desarrollo de ese país, considerando de modo particular los casos de Taiwán y Polonia. En la misma dirección, Rosales Sandoval (2009) aborda el tema de las remesas, al igual que López-Córdoba y Olmedo (2007), Tedesco (2008), Cerezo de Diego (2011), etc.

En lo que hace específicamente a la diáspora india, cabe señalar que existen numerosos estudios que la abordan desde distintas perspectivas $^{1}$ (Mansigh y Ajai, 1976; Palak-

$1 \quad$ Un mayor detalle sobre tales estudios puede consultarse en Rodríguez de la Vega y Meza (2014). 
shappa, 1980; Qureshi, 1984; Leonard, 1989; Motwani, Gosine y Barot-Motwani, 1993; Vertovec, 1995; Jayaram, 1998; Singh García, 2000; Chaulia, 2002; Khadria, 2006; Oonk, 2007; Lecrec, 2008; Puroshotaman, 2010; Therwath, 2010; Amrute, 2012, etc.). En cuanto a su abordaje en el marco de las relaciones internacionales, pueden mencionarse los trabajos de Khanna (2005), Rodríguez de la Vega (2006), Hymans (2009), Kapur (2010), Vardarajan (2010), Leclerc (2011), Mahalingam (2013), Murti (2013), Murti y Zaharna (2014), y otros.

\section{NOCIONES CONSIDERADAS}

Mientras Sheffer (1986) apunta que "las diásporas modernas son grupos étnicos minoritarios de origen migrante, que residen y actúan en los países de residencia, manteniendo un fuerte apego sentimental y material con sus países de origen” (p. 3), Vertovec señala que el término diáspora es usado en la actualidad para describir prácticamente cualquier población considerada "desterritorializada" o "transnacional", y Cohen indica que puede encontrárselo ya en el Deuteronomio, relacionado con la dispersión forzosa en el contexto de la historia religiosa judeo-cristiana (Vertovec y Cohen, 1999).

Como señalan Shain y Barth (2003), las diásporas pueden operar como lobbies étnicos en países de residencia y actuar como defensores de políticas exteriores multiculturales al tiempo que servir como conductos políticos para conflictos e intervención. Por ello, y por su carácter permanente en el sistema de
naciones-Estados, han empezado a recibir más atención.

El fenómeno migratorio en general guarda una estrecha relación con la cultura y la identidad. Respecto a la primera, desde otra disciplina de las ciencias sociales, Dube (1999) señala que la misma tiene que entenderse como un elemento esencial en la producción y reproducción cotidiana de la vida social, es decir, como las actitudes, normas y prácticas simbólicas y estructuradas por las que se perciben, articulan y experimentan las relaciones sociales, definiendo de esta manera la cultura en y por esas relaciones, que se predican al poder. En cuanto a la identidad, ella es entendida por Giménez (2007) como la dimensión simbólico-expresiva de todas las prácticas sociales, no como algo esencial sino de carácter intersubjetivo y relacional, generada en las interacciones cotidianas de los sujetos, lo cual les permite delimitar aquello que consideran "propio" de lo que no lo es, siendo la cultura subjetivada la matriz de las identidades sociales.

Ya en el marco de las relaciones internacionales, se considera el aporte de la propuesta de Shain y Barth (2003), acerca de que los enfoques constructivista y liberal comparten un espacio teórico que, aunque en distintos grados, reconoce el impacto de la identidad y la interacción doméstica en el comportamiento internacional, lo que resulta de utilidad en el abordaje de esta temática. Aunque relativamente reciente entre los enfoques teóricos de las relaciones internacionales, el constructivismo postula dos cuestiones básicas: a) que las estructuras fundamentales de la política internacional son sociales (socialmente cons- 
truidas), en vez de estrictamente materiales, $y$ b) que ellas modelan las identidades y los intereses de los actores, más que solo su conducta (Wendt, 1995). De igual manera, el estudio de redes étnicas requiere la consideración de la capacidad y disposición a cooperar y el rescate de la interdependencia de los actores. El liberalismo plantea que el Estado no es un actor independiente y rescata los actores individuales y grupos privados como actores en la política internacional. Los autores sugieren que el estudio de las diásporas con este marco teórico enriquece además el enfoque liberal en tanto permite expandir el significado del término doméstico.

Las diásporas, al igual que otros actores transnacionales, ejercen influencia tanto en el país de origen como en el de residencia, afectando a menudo al primero por la misma influencia que ejercen en el segundo. Como todo grupo de interés, utilizan todos sus recursos para la consecución de sus intereses (a través de remesas, donaciones, apoyo a candidatos políticos y partidos, proveedores de información acerca del impacto internacional de la política exterior, actores que sirven de herramientas de influencia sobre gobiernos extranjeros, etc.). Es en esta perspectiva en que se hace presente la consideración del poder blando, que describe la capacidad de un actor político para incidir en las acciones o los intereses de otros actores, utilizando medios culturales e ideológicos, complementándose a través de medios diplomáticos y enfatizando en las posibilidades de cooperación (Nye, 2004). De igual modo, se toma en cuenta la diplomacia pública, que en un sentido general alude a la comunicación con públi- cos foráneos para establecer un diálogo que permita informar al tiempo que influenciar (dado que esos públicos pueden influenciar en los intereses propios/nacionales); al respecto pueden darse dos tipos dentro de la misma: la diplomacia pública "gente a gente" y "gobierno a gente" (Mannheim, 1994 citado en Noya, 2006).

\section{LA DIÁSPORA INDIA}

De acuerdo con lo seńalado por Leclerc (2004), el uso de la idea de una "diáspora" para aludir a los emigrados de la India aparece de modo no explícito en 1976, en un texto de Bharati; el término se populariza en la década de los noventa, potenciado por el Comité de Alto Nivel sobre la Diáspora India, creado por el Gobierno de la India, que realizó un relevamiento de las comunidades emigradas, categorizando a los sujetos al interior de la misma como Indian Citizens not Residing in India (NRI) y Persons of Indian Origin (PIO), personas de origen indio que han tomado otra nacionalidad y las personas de origen indio que legal y técnicamente pueden calificarse como personas apátridas de origen indio, atendiendo a que no poseen documentación que acredite su origen (Sharma, s.f.). Por su parte, el Gobierno indio considera la "diáspora india" (2002) como "un término genérico que describe personas emigradas de territorios que están actualmente dentro de los límites de la República de la India”. Se refiere también a sus "descendientes" y señala que estas personas "residentes en tierras lejanas [..] retuvieron su ligazón emocional, cultural y espiritual con su país de origen" (The Indian Diaspora, párr. 1 
y 2$)^{2}$. Tal definición, considera que los emigrados han mantenido su identidad india (sin realizar más precisiones acerca de la misma) y, al mismo tiempo, establece una continuidad espacio/temporal entre el país de origen (India) y los distintos lugares de asentamiento.

En línea con lo señalado, se realizaron conferencias internacionales que abordaron la temática, antecedidas por la creación de un Centro para el estudio de esta diáspora, en la Universidad de Hyderabad (India), en 1996.

En ese marco, suelen distinguirse tres oleadas migratorias principales en términos de la evolución histórica de la migración desde la India: a) la emigración iniciada alrededor de 1830, bajo el colonialismo europeo, dirigida hacia distintas colonias -fase crucial en la emigración de la India-, b) la emigración hacia los países desarrollados industrializados, durante el periodo de posguerra de la Segunda Guerra Mundial (especialmente Estados Unidos, Canadá e Inglaterra), y c) la emigración reciente a Asia occidental (relacionada sobre todo con el trabajo en la industria del petróleo y fundamentalmente masculina) (Bhat, 1998).

\section{PRESENCIA DE LA DIÁSPORA INDIA EN AMÉRICA}

De acuerdo con lo mencionado por el Comité de Alto Nivel sobre la Diáspora India, dicha diáspora posee un volumen de más de veinte millones de indios que residen fuera de su país de origen (algunos autores ya hablan de 25 millones), incluyendo las distintas categorías mencionadas, asentados en más de setenta países (MEA, 2002).

Respecto a los emigrados de la India en relación con el continente americano, cabe seńalar que en la mayoría de los casos su llegada se produjo durante el siglo XLX, y continúa hasta el presente, en que se han facilitado tanto las comunicaciones como las posibilidades para viajar. Al considerar el volumen de la población de ese origen en América en general, se aprecia que la mayoría se encuentra fundamentalmente en países de habla inglesa: Canadá, donde superan 1,2 millones de personas, siendo el tercer grupo inmigrante en volumen (Government of Canada, 2013); Estados Unidos, con 2,81 millones de personas de origen indio (us Census, 2010), siendo también el tercer grupo inmigrante allí; Trinidad y Tobago, donde según los datos de su censo 2011, la población de origen indio constituye el 35,4\% de la población total del país (Government of the Republic of Trinidad and Tobago, 2012); Suriname, en donde el censo de 2012, arrojó que la población de origen indio allí es de 148.443 personas y constituye el $27,4 \%$ del total (General Statistics Bureau of Suriname, 2012); la República de Guyana, cuyo su censo de 2002, señala que este grupo constituye el $43,5 \%$ del total de su población (Guyana

\footnotetext{
2 Se concuerda con el planteo de Oonk (2007), acerca de que el hecho de hablar sobre la "diáspora india" supone una mirada crítica de ambos términos, cuestionando a qué refieren, el tipo de unidad que suponen y por quién son enunciados.
} 
Bureau of Statistics, 2002) ${ }^{3}$; Belice, donde los resultados de su censo de 2010, indican que la población de este origen representa el 1,1\% de su población (The Statistical Institute of Belize, 2013), y Jamaica, que de acuerdo con el reporte de MEA (2002) alberga alrededor de 60.000 descendientes de población de origen indio.

En cuanto a los países de América Latina, Puerto Rico no reporta en su censo de 2010 población de origen indio (León López, 2013) -aunque el reporte de MEA (2002) plantea la existencia de aproximadamente 8000 personas de origen indio allí-; igual sucede con México (INEGI, 2010), donde la Embajada de la India en México y Belice, señala que la comunidad de origen indio allí es de no más de 2000 personas (abarcando tanto NRI como PIO). En Guatemala no se encuentran reportes de la presencia de inmigración de origen indio (огм, 2013), sin embargo, un informe de orm (2011) señala que los indios están exentos de visa para ingresar a este país, de modo que algunos, tomando esa ventaja, se mezclan con ciudadanos centroamericanos para ingresar a México por Tapachula, Chiapas, y de allí poder ir hacia Estados Unidos. En el caso de El Salvador, su vi Censo de Población y vi de Vivienda, 2007, señalan la presencia de 10 indios sobre los 574 inmigrantes de origen asiático que residen en ese país (Ministerio de Economía, 2009). En Honduras, de acuerdo con la información provista por el censo de 2001, no se halló mención desagregada a inmigración proveniente de la India (solo se aclara "Otros países") (огм, 2001). Por su parte, en Costa Rica se encuentran fundamentalmente inmigrantes latinoamericanos y norteamericanos, y se menciona un porcentaje de $12 \%$ caracterizado como "Otros" (Dirección General de Migración y Extranjería, 2012). Tampoco se encontraron datos de inmigrantes indios en la información migratoria de Nicaragua (oIM, 2012). En cuanto a Panamá, Lizcano Fernández (2005) señala un 4\% de asiáticos en su población (chinos e indios). El reporte de MEA (2002) señala la presencia de 40.000 personas de este origen en la isla de Guadalupe, región francesa de ultramar, habiéndose ido varios de ellos a la Guayana Francesa y siendo en general parte de la diáspora Tamil ${ }^{4}$. En cuanto a Cuba, su censo de 2012 desagrega información en función de su población blanca, negra y mestiza o mulata, sin ofrecer datos de inmigración india o descendientes (Oficina Nacional de Estadística e Información, 2012). Por su parte, la Embajada de la India en Venezuela (2012) señala la presencia de 50 familias de origen indio en ese país, con 35 de ellas asentadas en Caracas. La Embajada de la India en Colombia (s. f.) comenta que la comunidad india en este país está conformada por alrededor de 250 personas, la mayoría de ellas profesionales

\footnotetext{
3 Su actual presidente, Donald Rabindernauth Ramaotar, acaba de ser premiado en la India por el primer ministro Narendra Modi, con ocasión del Pravasi Bharatiya Diwas, en que se premia a miembros de la diáspora por sus aportes en distintos campos (Grupo de Trabajo sobre India, Comité de Asuntos Asiáticos, CARI, a publicarse en febrero 2015).

4 Pueden consultarse más detalles de la población de origen indio en zonas de habla francesa en América, en el reporte de MEA (2002).
} 
de empresas multinacionales; en Ecuador, la Embajada señala la existencia de una pequeńa comunidad de aproximadamente 60 personas, que laboran en pequeñas empresas y en el sector de servicios; el informe de la orm (2012b) señala que entre los nacionales de otros países llegados recientemente a Ecuador por país de origen y sexo (2010), había 139 hombres y 53 mujeres originarios de la India (junto a otros inmigrantes de Bangladesh, Nepal y Pakistán). Por su parte, la Embajada de la India en Brasil (s. f.) sostiene que la comunidad en ese país es pequeña (alrededor de 2000 personas), asentadas principalmente en São Paulo, Rio de Janeiro y Manaos, mientras que la Embajada de India en Perú y Bolivia (2014) señala la existencia de una comunidad pequeña en Perú, de alrededor de 400 personas de este origen, que trabajan en actividades comerciales (señalando también la llegada de profesionales indios que arribaron con las compañías de ese origen y también indios que trabajan con los Misioneros de la Caridad y otras organizaciones cristianas asentadas en Lima, Puno y Chimbote), al igual que en Bolivia, donde se reportan 180 personas de ese origen que trabajan en comercio, transporte, agricultura, entre otros. La información provista por la Embajada de la India en Chile (s.f.), por su parte, da cuenta de una comunidad de alrededor de 1500 personas, en su mayoría sindhis, asentadas sobre todo en el puerto de Iquique, en Santiago y Punta Arenas. En lo que se refiere a Uruguay, la Embajada de la India en Argentina, concurrente en Paraguay y Uruguay (s. f.), comenta la existencia de entre 60 y 100 personas, mayormente empleadas en empresas relacionadas con el sector de tecnologías y residentes en Montevideo ${ }^{5}$; de igual modo, se menciona que algunos de ellos pertenecen a las comunidades de gujaratis y sindhis, dedicándose a la importación de elementos de la India. Con respecto a Paraguay, se señala también la presencia de alrededor de 100 personas de origen indio, gujaratis y sindhis, residentes en Ciudad del Este. De igual modo, se señala la presencia de una comunidad de aproximadamente 2000 personas de origen indio en Argentina, con alrededor de 200 de ellos, de primera generación, residentes en Buenos Aires, y otros 300, aproximadamente, en el norte del país, sobre todo sindhis y sikhs, empleados de distintas empresas o cuentapropistas.

\section{DINÁMICAS EN LA RELACIÓN TRIÁDICA: PAÍS DE ORIGEN-DIÁSPORA-PAÍS DE RESIDENCIA}

\section{LAS POLÍTICAS DEL PAÍS DE ORIGEN}

Hasta la independencia india, los emigrados eran vistos como parte del futuro del país, siendo Gandhi mismo un emigrado en Sudáfrica, el cual retornó luego a la India. Podía entonces verse al Partido del Congreso defendiendo los intereses de los emigrados ante diversas situaciones que lo requerían (Sinha-Kerkhoff y Bal,

\footnotetext{
La emigración de la India en Uruguay está siendo abordada actualmente por un proyecto de investigación de la autora, desarrollado en el marco del Área de Asia y África, Unicom, Facultad de Ciencias Sociales, Universidad Nacional de Lomas de Zamora, provincia de Buenos Aires, República Argentina.
} 
2003). Nehru, por su parte, no cultivó esos lazos porque creía que los emigrados indios habían perdido su ciudadanía e identidad al migrar del país, y que no precisaban el apoyo del mismo, y en razón de una política de no intervención en otros Estados. Los emigrados poseían en ese momento tres opciones: optar por la ciudadanía británica, por la india o por la del lugar de residencia; se les recomendó adquirir la ciudadanía local y trabajar por la independencia en su lugar de residencia (Lal, 2001, citado en Nijhawan, 2003) ${ }^{6}$. El gobierno Janata, en 1977, inició cambios favorables hacia los emigrados, continuados por la gestión de Rajiv Gandhi, luego de acceder al poder, en 1984. Sin embargo, el mayor cambio se daría junto a las reformas económicas de liberalización de la década de los noventa, en las políticas implementadas por la gestión de Atal Bihari Vajpayee (Partido Bharatiya Janata), que se han ido profundizando y continúan hasta el presente (Leclerc, 2004; Hercog y Siegel, 2010). El Gobierno indio desarrolló distintas acciones en torno a la diáspora, entre ellas cabe destacar la puesta en marcha del Plan de Credenciales para las PIO, que consideraba todos los segmentos de la diáspora (1999): visa de largo tiempo (20 años), que permite a los PIO poseer propiedades y acceder al sistema educativo en India (Chemouni, 2009); la creación de una división de NRI y PIO, perteneciente al Ministerio de Asuntos Exteriores, presidida por un secretario; la creación de un Comité de Alto Nivel sobre la Diáspora India, que realizó un informe exhaustivo sobre la misma y su relación con India, concluyendo con distintas recomendaciones (2000); la creación del Pravasi Bharatiya Divas (Día de la Diáspora India) y del premio Pravasi Bharatya Samman, que busca distinguir a los emigrados más exitosos y destacados; el establecimiento de una cuasi doble ciudadanía para algunos países de residencia, vigente desde 2003, que permite a sus beneficiarios invertir en la industria y en la agricultura, adquirir propiedades, enviar a sus hijos a escuelas y universidades, sin derecho a votar o a postularse para la función pública (The Indian Diaspora, s. f.; Chemouni, 2009); la posterior creación del Ministerio para los Asuntos de los Indios en el Extranjero (MOIA), a cargo de un ministro autónomo, al tiempo que una estructura institucional que colabora en la implementación de la tarea del ministro: a) El Centro de Facilitación de los Indios de Ultramar, b) El Consejo Indio de Empleo de Ultramar, c) El Consejo Asesor Global de Personas de Origen Indio, en la Oficina del Primer Ministro, d) La Fundación India para el Desarrollo de los Indios de Ultramar, e) La Red Global India de Conocimiento y f) Centros de los Indios de Ultramar (establecidos en Washington y Abu Dhabi para proveer información sobre temáticas relativas a los emigrados). A la par de ello, India ha desarrollado diversos programas focalizados en la diáspora, entre los cuales pueden mencionarse el Programa de Becas para los Niños de la Diáspora (SPDC) que ofrece becas de grado en

\footnotetext{
6 Sin embargo, Nehru sostenía al mismo tiempo: "Wherever in this world there goes an Indian, there is also a bit of India with him" (Nehru citado en Sinha-Kerkhoff y Bal, 2003, p. 15).
} 
universidades indias, el Programa "Conoce India”, etc. (Ashraf, 2005; Chemouni, 2009; Hercog y Siegel, 2010).

Las reformas en la administración del fenómeno migratorio se dan en los planos nacional, bilateral y multilateral. En ese marco, puede señalarse la creación de instancias institucionales tales como el Consejo Indio de Empleo para los Emigrantes, el Centro de Recursos para Trabajadores Emigrados, el Protector General de Emigrantes, el Fondos para el Bienestar de la Comunidad India, un Centro de Recursos para Migrantes (Kochi, Kerala), etc. También se establecieron tratados bilaterales con distintos países (Qatar, en 1985; Jordania, en 1988; Emiratos Árabes Unidos, en 2006; Kuwait, en 2007; Omán, en 2008 y Malasia y Bahrain, en 2009) y se firmó un Memorando de Entendimiento sobre Asociación concerniente a Movilidad Laboral, con Dinamarca, a la espera de desarrollar acuerdos semejantes con otros países europeos y surasiáticos. En 2006, se firmó un acuerdo bilateral de seguridad social con Bélgica, continuando luego con otros países (Francia, Alemania, Suiza, Luxemburgo, Países Bajos, Hungría, Dinamarca, la República Checa, República de Corea y Noruega), e iniciándose encuentros para avanzar en igual dirección con Canadá, el Reino Unido y Estados Unidos (Ministry of Overseas Indian Affairs, s. f.).

En el plano multilateral, la India participa del Proceso Colombo, apoyado por la Organización Internacional para las Migraciones (оIM), con la cual se firmó un Memorando de Entendimiento, en el que se acuerdan condiciones para la mejor administración de empleo legal de trabajadores indios en la Unión Euro- pea. Existe también, desde 2000, un Grupo de trabajo conjunto India-Unión Europea sobre asuntos consulares, y se adoptó, en 2005, un Plan Estratégico de Acción Conjunta. India es también miembro del Encuentro Asia-Europa (ASEM) (ASEMINFOBOARD, s.f.), y forma parte de otras instancias como el Foro Global sobre Migración y Desarrollo (GFMD) (Hercog y Siegler, 2010). Paralelo a las acciones desarrolladas por el Gobierno nacional, estados como Gujarat, Kerala y Punjab, crearon instituciones específicas referidas a la temática de los emigrados (Chemouni, 2009).

En enero de 2015, el Gobierno indio informó a la Suprema Corte su decisión de aceptar la recomendación de la Comisión Electoral sobre permitir a los indios no residentes votar desde el exterior a través de votación por correo o electrónica, concediéndole la Justicia ocho semanas al Gobierno para realizar las enmiendas necesarias a la ley, que permitan la implementación de tal decisión (Rajagopal, 2015; Mahapatra, 2015). La medida va en línea con la creciente importancia que la gestión del país de origen le asigna a la diáspora.

\section{LAS DINÁMICAS DE LA DIÁSPORA}

En paralelo a las acciones que llevara adelante el Gobierno indio, acerca de las políticas progresivamente desarrolladas hacia ella, la diáspora, al tiempo que alcanzaba distintos destinos, fue también organizándose en diversas redes creadas de manera voluntaria. Entre esas organizaciones puede mencionarse la Global Organization of People of Indian Origin (GOPIO), que cuenta con una casa central en Nueva York y otra en las islas Mauricio, cuyo 
primer encuentro se llevó a cabo en 1989, con el objeto de reunir a la diáspora, y salvaguardar y promover sus intereses.

Aparte de la Gopio, en la actualidad pueden encontrarse distintas asociaciones desarrolladas con base en identidades regionales, de religión, casta ${ }^{7}$, etc., entre otras la World Telugu Federation (WTF), surgida en 1992 como ONG, para la promoción del lenguaje y la cultura telegu entre estos emigrados; la Confederación Mundial Tamil, establecida en 2002, en una de las conferencias de estudios tamiles que esta comunidad organiza (con equivalentes regionales tales como la Federation of Tamil Sangams of North America y la Australian Federation of Tamil Sangams), la Vishwa Gujarati Samaj, organización internacional para la comunidad gujarati, establecida en 1989 para promover la cultura y los intereses de la comunidad, que viene organizando conferencias mundiales (contando con el apoyo del Gobierno de Gujarat). Asimismo, las personas de habla bhojpuri están organizadas y realizan encuentros mundiales, para ello cuentan con el apoyo del gobierno de Bihar y, aunque desarrollaron distintos sitios web dedicados a esta cultura particular, planean un website para Vishwa Bhojpuri Sammellan, al tiempo que existe también la Bhojpuri Association of North America (BANA).

De igual modo, las identidades religiosas han derivado en distintas agrupaciones que reúnen y promueven esos elementos culturales, entre ellas la World Sikh Organization, la International Sikh Youth Federation, la American Federation of Muslims of Indian Origin, la Vishwa Hindu Parishad, etc. ${ }^{8}$ (Bhat y Narayan, 2010).

Más allá de los espacios de asociación e institucionalización de los distintos segmentos de la diáspora, un tema crucial es el de la remisión de remesas a la India, que en 20122013 fue de 67.600 millones (The Economist, 2014). De igual relevancia resultan la inversión extranjera directa, la transferencia de conocimiento de emigrados que se asentaron definitivamente en otro país o tras el retorno de los mismos, la colaboración de los emigrados no solo con remesas, sino con espacios de aportes económicos como donaciones, que contribuyen al desarrollo de áreas y grupos específicos del país de origen, etc.

\section{LA MIGRACIÓN INDIA EN ESTADOS UNIDOS Y SU ACTIVIDAD EN RELACIÓN CON EL PAÍS DE ORIGEN Y EL DE RESIDENCIA}

Los emigrados indios en Estados Unidos llegaron hacia finales del siglo XLX e inicios del $\mathrm{xx}$, siendo los primeros inmigrantes allí, procedentes de Punjab, que se asentaron en la costa oeste del país.

Tras su arribo al país, fueron objeto de campañas racistas y distintas restricciones que

\footnotetext{
Tales desarrollos abren paso a la consideración de la dinámica de las identidades nacionales y el papel de las diásporas en ella.

8 La existencia de movimientos religiosos transnacionales, originados en la India, ha abierto el debate acerca de la "orientalización de Occidente" y el efecto de Occidente en los desarrollos de tales grupos en la India misma, entre otras temáticas de interés.
} 
les imposibilitaron la compra/propiedad de tierras y la adquisición de la nacionalidad estadounidense; sin embargo, la pos Segunda Guerra Mundial, los encontró con una merma de restricciones hacia los asiáticos -entre ellos, los indios- y en 1946 se dio una enmienda que estableció una cuota de arribos para los inmigrantes de este origen, haciéndolos elegibles para la naturalización (Luce-Cellar Bill). En 1965, expidió el Acta de Servicios de Nacionalidad e Inmigración, que eliminó anteriores restricciones (desestimando el sistema de cuotas para ingreso y dando preferencia a quienes ya tenían familiares que residían en el país) y facilitó la inmigración de profesionales indios.

La participación política de la comunidad en / desde Estados Unidos estuvo asociada a la posibilidad de naturalizarse o nacionalizarse allí, posibilidad abierta en 1946. Diversos trabajos señalan que hubo un marcado nacionalismo entre los inmigrantes indios allí asentados, estimulado, en los primeros años de asentamiento, por la lucha independentista india contra el imperio británico, registrándose en ese escenario el surgimiento del movimiento político Gadar, prorevolucionario. Aparte de ello, progresivamente se dio un proceso de asociación e institucionalización, evidenciado en el surgimiento de organizaciones como la Sociedad Khalsa Divan de la Costa del Pacífico (1915), la Liga Normativa India de América (1918), la Sociedad Hindustani de América para el Bienestar y Reforma (1920), la Sociedad Hindustan de América, la Asociación India de América, la Liga India de Bienestar y la Liga India de América. Ese desarrollo continuaría posteriormente, tras la independencia de la India, pero con la atención puesta en la inclusión en el país de residencia.

Ya entonces puede rastrearse el eco de situaciones del país de origen en la diáspora cuando, por ejemplo, se creó la Liga India de América en 1972, un año después del surgimiento de la Liga Pakistaní de América. En igual dirección, la Asociación de Indios de América (creada en 1967), realizó una campaña para obtener una clasificación separada para los indios en el censo, a diferencia de los pakistaníes, que seguían agrupados, con otros colectivos, bajo el rótulo "asiáticos" (Mohammad-Arif, 2000). Otras organizaciones, se propusieron crear conciencia acerca de la participación política en el país de residencia, tales como el Comité de Acción Política India-América, el Consejo de la Amistad India Americana, el Foro Indio Americano de Política Educacional, el Centro Exterior Indio para la Conciencia Política, la Asociación de dueños de hoteles asiático americanos, etc. De igual modo, se formaron la Federación de Asociaciones Indo Americanas y la Asociación Nacional de Descendientes de Asiático-indios, en atención a diversos reclamos sobre la falta de unidad entre las asociaciones comunitarias indias. Algunas de ellas avanzaron en la organización de diversas acciones concernientes a asuntos indios o indo americanos, como por ejemplo, la Federación Nacional de Asociaciones Indo Americanas y la Asociación Americana de Médicos de origen indio, que hicieron campaña para evitar los recortes de los fondos de Medicare a los hospitales que empleaban doctores formados en el exterior. En igual sentido, la Federación persuadió al Congreso estadounidense de no 
vender sistemas de control y alerta aéreos a Pakistán (Mishra y Mohapatra, 2002, citados por Janardhanan, 2013). Asimismo, distintos trabajos (Therwath, 2007, 2012, entre otros) señalan la presencia de acción política de hindutva $a^{9}$ en algunos sectores de la comunidad, evidenciada, por ejemplo, en las divisiones experimentadas en los miembros de la Asociación de dueños de hoteles asiáticoamericanos, ante la negación de visa a quien por entonces era la máxima autoridad política de Gujarat, Narendra Modi (actualmente primer ministro de la India), atendiendo a los episodios ya conocidos (Sirohi, 2005, citado por Janadharnan, 2013), entre otros.

Por lo demás, la comunidad ha participado activamente en los últimos treinta años en las elecciones en Estados Unidos. Dalip Singh Saund fue el primer asiático electo como congresista (por California, en 1956, por el partido demócrata); Bobby Jindal fue candidato a gobernador por Louisiana, en 2003, aunque perdió, y en 2004 llegó a la Cámara de Representantes (y fue reelecto en 2006, por el partido republicano), siendo actualmente gobernador del estado de Louisiana; en 2012, Ami Bera (Amerish Babulal) se convirtió en el tercer indio americano electo en el Congreso norteamericano (por el partido demócrata). Por su parte, Nikki Randhawa Haley es gobernadora de Carolina del Sur (por el partido republicano), Satveer Chaudhary fue senador en el Estado de Minnesota, Nimi McConigley (Nirmala Swamidoss McConigley) fue legisladora en la legislatura de Wyoming ${ }^{10}$ y Kumar P. Barve fue líder de la mayoría en la legislatura de Maryland y continúa siendo miembro de ella en la actualidad (por el partido demócrata). La comunidad india hasta el momento ha participado en la colecta de fondos y la creación de grupos de apoyo a candidatos específicos. También en 1993, se formó el Comité Bipartidario del Congreso, de India y de los representantes indoamericanos ${ }^{11}$. Ese comité alcanzó 164 miembros en diez años, y sirvió de espacio de lobby a la comunidad india en el país sin tener un candidato de ese origen hasta 2004 -con la llegada de Bobby Jindal-, logrando ejercer influencia en distintos asuntos, especialmente algunos que hacen a la política exterior de Estados Unidos. Sus miembros han trabajado por la visita de Clinton a India y la visita del primer ministro Atal Bihari Vajpayee a Estados Unidos. En 2004, se formó un comité bipartidario en el Senado, similar al Comité de India, denominado "Amigos de India" 12 , coordinado por el Comité de Acción Política Estados Unidos-India (UsINPAC). Estos Comités trabajaron también en el acuerdo de energía nuclear para uso civil entre ambos países ${ }^{13}$.

Además de destacar el accionar de la comunidad india asentada en Estados Unidos

9 El uso del término hindutva remite en este caso a la ideología del nacionalismo hindú.

10 Nacida en Madras (India), fue la primera legisladora nacida en la India en servir como legislador en ese país.

11 Frank Pallone fue su primer presidente, por los demócratas, junto a Bill McCollum, por los republicanos.

12 Sus primeros presidentes fueron Hillary Clinton, por los demócratas, y John Cornyn, por los republicanos.

13 Cabe recordar aquí que el aumento poblacional y de actividad económica hacen que India tenga en perspectiva el 
(sin suponer por ello homogeneidad en su ideario, accionar o participación), cabe señalar que en el presente, el volumen de su población es de 2,81 millones de personas (primera y segunda generación), siendo, como ya se apuntó, el tercer grupo migrante más extenso asentado en ese país. Su población es comparativamente joven, con altos niveles de logros académicos y estándares económicos ${ }^{14}$. Los inmigrantes de origen indio constituyen el grupo extranjero más beneficiado por el programa de visas $\mathrm{H}$ 1B (que ha sido motivo de diálogo entre ambos Gobiernos, ante la consideración de una reforma migratoria en Estados Unidos) ${ }^{15}$, y constituyen porcentajes sustanciales de las visas H-4, L-1 y L-2, etc. En 2012, esta comunidad contribuyó con 13 mil millones a las remesas de dinero que India recibió ese año, siendo el segundo país en lo que hace a envíos netos de dinero y como destino de los emigrados indios, luego de los Emiratos Árabes Unidos en ambas temáticas (MPI, 2014).

Con todo lo señalado, cabe decir que el alcance del accionar de la comunidad india en Estados Unidos es todavía limitado, en atención al pequeño volumen de sus votantes, por lo que precisa profundizar su accionar en el Congreso del país de residencia. Por otro lado, autores como Aiyar (2012), destacan el accionar de los individuos y también los agentes económicos indios y, en este caso, norteamericanos con intereses en la India ${ }^{16}$. La presencia de la diáspora y su capacidad de acción se hicieron evidentes en la reciente visita del primer ministro Modi a Estados Unidos, con manifestaciones a favor pero también algunas otras que lo cuestionaron (Grupo de Trabajo sobre India, 2014).

\section{A MODO DE CIERRE}

Existe en la actualidad una presencia nutrida de actores no estatales en la política internacional, entre ellos las diásporas. En ese marco, en el tratamiento de la cuestión migratoria, el paso en la gestión india, de una concepción nehruviana, más asociada a lo territorial, a otra más ligada a la etnicidad (del partido Bharatiya Janata), ha llegado a materializarse en una compleja estructura desarrollada por India (incluyendo el establecimiento del Ministerio para los Asuntos de los Indios en el Extranjero) para profundizar sus lazos con la diáspora ${ }^{17}$. El desarrollo de esa gestión, por otro lado, alude a

\footnotetext{
incremento de sus requerimientos de provisión energética y la dependencia de la importación de energía somete al país a circunstancias que desea evitar. En ese marco, el aprovechamiento de infraestructura y habilidades ya desarrolladas en el campo de la energía nuclear, la vuelve una opción de interés y, en tal sentido, India ha realizado ya numerosos acuerdos para la provision de uranio, reactores nucleares y tecnología nuclear (Grupo de Trabajo sobre India, 2015b).

14 La pobreza dentro de la comunidad aqueja a un porcentaje pequeño de la misma (MPI, 2014).

15 En 2012, el 64\% de las visas de este tipo concedidas, lo fueron para migrantes de origen indio (MPI, 2014).

16 Pueden consultarse más datos sobre la temática, en Rodríguez de la Vega (2014), actualmente en edición para publicación.

17 India ha desarrollado también una estructura digital para profundizar el contacto con su diáspora, como parte de su diplomacia pública (Murti y Zaharna, 2014).
} 
un nacionalismo de larga distancia o diaspórico para movilizar a los emigrados, que supone distintas acciones desde el Estado indio, para operar sobre la identidad india en el ámbito transnacional.

Al mismo tiempo, la diáspora devino en un factor de autorreferencia, evidenciado en las distintas instituciones desarrolladas en su ámbito, algunas basadas en identidades regionales indias (por ejemplo, distintas organizaciones globales formadas por inmigrantes de Tamil Nadu, Gujarat, Andhra Pradesh, Bhojpuri, etc.) y otras en una identidad panindia -como la Global Organization of the People of Indian Origin (GopIO), la más significativa entre los emigrados indios- (Bhat y Narayan, 2010). Tales organizaciones y su estructuración en función de las identidades mencionadas, han fortalecido y profundizado la relación con el Gobierno nacional del país de origen, así como con gobiernos estaduales al interior de India, al tiempo que proyectado y multiplicado el poder de tales identidades culturales como factor de cohesión en distintos segmentos de la diáspora y proyección de imágenes de esos grupos y, por tanto, de culturas de la India en el exterior del país.

Mientras el Estado indio potencia los canales relacionados con el uso de su poder blando ${ }^{18}$ y la "diplomacia diaspórica", se dan fenómenos paralelos que impulsan tales acciones, tales como una mayor conciencia de la población india acerca de la política exterior del país ${ }^{19}$. Al respecto, Hall (2012) comenta que la nueva diplomacia pública india busca nuevas audiencias en India, en Occidente y en el mundo en desarrollo; que parece haber un intento democratizador en la hechura de la política exterior-materializado a través del diálogo con comunidades fuera de Nueva Delhi y la élite diplomática-, y agrega que India avanza en el uso de nuevas tecnologías de la comunicación para alcanzar los distintos públicos objetivo (en tal sentido, tanto la estructura digital desarrollada para "alcanzar" la diáspora india como el despliegue comunicacional del primer ministro Modi, con alcance dentro y fuera de India, parecen ser dos claros ejemplos).

En el caso específico del segmento de la diáspora asentado en Estados Unidos, distintas acciones evidencian la ligazón de la esfera política internacional con la doméstica (del país de origen) y su promoción de los intereses de la India como grupo de presión, por ejemplo, su apoyo para la realización del encuentro entre India y Estados Unidos para el logro de un acuerdo sobre energía nuclear para uso civil ${ }^{20}$, la proyección de la presencia

18 Thussu (2014) aborda el soft power en relación con la industria del cine en hindi, conocida popularmente como "Bollywood", que permanece como una manifestación prominente de contenido indio en los medios globales y representa una industria de 3,5 mil millones de dólares.

19 Al respecto, Srivastava y Singh (2014) abordan la cuestión de la diplomacia pública y la serie de Charlas Distinguidas sobre Política Exterior de la India, iniciadas en 2010.

20 En el marco del anuncio compartido de la Cooperación Nuclear Civil (junio de 2005), India accedió a separar los medios nucleares civiles y militares, y a tener los primeros bajo las normas de seguridad de la International Atomic Energy Agency (IAEA), obteniendo reconocimiento nuclear de hecho. El acuerdo fue aprobado por la IAEA en 2008, y 
de hindutva y las consecuentes divisiones al interior de la comunidad emigrada -que traducen un debate del país de origen-, la plena organización de la llegada y el desarrollo de la visita del primer ministro Modi a Estados Unidos y del presidente Obama a la India ${ }^{21}$, la creación de comités bipartidarios en ambas cámaras del Congreso norteamericano, etc. De igual manera, el reciente anuncio de la habilitación para votar desde fuera del país, siendo emigrado, se inserta en esa lógica de modelado permanente y mutuo de los distintos segmentos que "portan" la(s) identidad(es) nacional(es). En línea con ello, cabe mencionar un elemento que hace a la política exterior del país de residencia, reflejado en el nombramiento como embajador norteamericano en India, de Richard Verma, primera persona de origen indio en desempeñar ese cargo (Grupo de Trabajo sobre India, 2015a), produciéndose aquí el encuentro coincidente de las estrategias de los distintos actores.

Probablemente, la potencialidad del rol de la diáspora para ambas sociedades (la del país de origen y la del país de residencia) haya quedado expresada, entre otros, en una declaración que hicieran el presidente Bill Clinton y el primer ministro Atal Bihari Vajpayee en marzo de 2000, titulado "Las relaciones IndiaEstados Unidos: una visión para el siglo XXL", en que se señala: "Nuestra sociedad [...] está reforzada por los lazos de becas, comercio y el creciente parentesco entre nuestra gente. La industria, empresa y contribuciones culturales de los americanos de herencia/origen indio han enriquecido y hecho más vivaces ambas sociedades" (Clinton y Vajpayee citados en Inderfurth, 2012).

Finalmente, cabe apuntar que la relación triádica que comprende al país de origen, la diáspora misma y sus países de residencia, mencionada por Sheffer (1986), evidencia dinámicas que articulan lo local y lo global -mutuamente constitutivos- de manera permanente, con una India inserta de este modo en las redes y los imaginarios transnacionales de la globalización como un espacio nacional expandido y con una continuidad histórica que abarca a todos los segmentos de su diáspora. Cabe ahora avanzar en delinear con más certeza los contornos de estas dinámicas en aquellos países de residencia en que la diáspora india no cuenta con poblaciones de gran volumen, tales como aquellos de América Latina.

\section{REFERENCIAS}

Adamson, F. B. (2006). Crossing Borders: International Migration and National Security. International Security, 31 (1), 165-199.

ese mismo año, el Grupo de Abastecedores Nucleares proveyó a la India de material para acceder a tecnología nuclear civil y combustible (Ogden, 2011).

21 En enero de 2015, durante la segunda visita del presidente Obama a la India -invitado de honor a la celebración del Día de la República-, se renovó la asociación de defensa entre India y Estados Unidos, y se superaron las diferencias sobre su acuerdo de cooperación en energía nuclear para usos pacíficos (Haidar, 2015). Estados Unidos anunció también su apoyo a la membresía de India en los cuatro regímenes nucleares (Grupo de Trabajo sobre India, Comité de Asuntos Asiáticos, 2015 a). 
Amrute, S. (2012). The New Non -Residents of India: A Short Hisotry of the NRI. En D'Costa, A. P. (ed.). A New India? Critical reflections in the Long Twentieh Century (pp. 127-150). UK y USA: Anthem Press.

Anderson, B. (1993). Comunidades Imaginadas. Reflexiones sobre el origen y la difusión del nacionalismo. México: Fondo de Cultura Económica.

ASEMINFOBOARd (s. f.). The Official Information Platform of The Asia - Europe Meeting. Recuperado de http://www.aseminfoboard.org/about-asemmenu.html

Ashraf, F. (2005). Indian Diaspora: Projecting Indian's image. Strategic Studies. Islamabad: Institute of Strategic Studies Islamabad (Iss). Recuperado de http://www.issi.org.pk/old-site/ss_Detail. php?dataId $=331$

Betts, A. (2009). Forced Migration and Global Politics. United Kingdom: Wiley-Blackwell.

Betts, A. (2014). International relations and Forced Migration. En Fiddian-Qasmiyeh, E., Loescher, G., Long, K. y Sigona, N. (eds.) (2014). The Oxford Handbook of Refugee and Forced Migration Studies (Chapter 6). Oxford: Oxford University Press.

Bhat, Ch. (1988). India and the Indian Diaspora. A Policy Issues. Ocassional Paper 4. Department of Sociology. University of Hyderabag. Recuperado de http://www.indiandiaspora.nis.in/

Bhat, Ch. y Narayan, K. L. (2010). Indian Diaspora, Globalization and Transnational Networks: The South African Context. J. Soc. Sci., 25 (1-2-3), 13-23.

Brochmann, G. y Hammar, T. (eds.) (1999). Mechanisms of Immigration Control. Oxford/New York: Berg.

Cerezo de Diego, P. (2011). Migraciones, desarrollo, remesas y crisis económica internacional. Anuario Jurídico y Económico Escurialense, XLIV, 187-208.
Chaulia, S. S. (2002). BJp, India's Foreign Policy and the "Realist Alternative" to the Nehruvian Tradition. International Politics, 39, pp. 215-234.

Chemouni, B. (2009). The Diaspora as an economic asset: How China and India use their diaspora to support their economic development [Tesis de grado. London School of Economics and Political Science]. Londres: Inglaterra.

Cornelius, W. A., Takeyuki T., Philip, L. M. y Hollifield, J. F. (eds.) (2004). Controlling immigration: a global perspective. Stanford: Stanford University Press.

Dannreuther, R. (2007). People on the Move: Migration as a Security Issue. En International Security: The Contemporary Agenda (pp. 100-117). Cambridge, MA: Polity Press.

Deudney, D. (1990). The Case Against Linking Environmental Degradation and National Security. Millennium: Journal of International Studies, 19 (3), 461-476.

Dirección General de Migración y Extranjería (2012). Migración e integración en Costa Rica. San José, Costa Rica: DGme. Recuperado de http://www. migracion.go.cr/integracion_desarrollo/Migracion $\% 20 \mathrm{e} \% 20$ Integracion $\% 20 \mathrm{en} \% 20$ Costa $\% 20$ Rica\%20Informe\%202012.pdf

Dube, S. (1999). Pasados Poscoloniales. México, D.F.: El Colegio de México.

Embajada de la India. Argentina. Concurrente en Paraguay y Uruguay (s.f.). Relaciones bilaterales India-Argentina. Recuperado de http://www.indembarg.org.ar/Bilateral\%20Brief\%20India $\% 20$ Argentina\%20Mar\%202013\%20(Spanish).pdf Embajada de la India. Brasil (s.f.). Relaciones India Brasil. Recuperado de http://indianembassy. org.br/bilateral/

Embajada de la India. Colombia y Ecuador (s.f.). rela- 
ciones bilaterales. Recuperado de http://www. embajadaindia.org/en/\#

Embajada de la India. Chile (s.f.). Comunidad india. Recuperado de http://www.embajadaindia.cl/ page/display/25/63

Embajada de la India. México y Belice (s.f.). Relaciones bilaterales. Recuperado de http://www.indembassy.org/eoi_ol.php?id=Bilateral

Embajada de India. Perú y Bolivia (2014). Relaciones bilaterales. Recuperado de http://www.indembassy. org.pe/english/bilateral-relation/india-peru.html

Embajada de la India. Venezuela (2012). Relaciones India-Venezuela. Recuperado de Recuperado de http://www.embindia.org/India-Venezuela_Rel. php

Frechette, A. (2004). Tibetans in nepal. The Dynamics of International Assitance among a Community in Exile. New York-Oxford: Berghahn-University of Oxford.

Geertz, C. (1963). The Integrative Revolution: Primordial Sentiments and Civil Politics in the New States. En Geertz, C. (ed.). Old Societies and New States: The Quest for Modernity in Asia and Africa (pp. 105-157). New York: The Free Press.

General Statistics Bureau of Suriname/Algemeen Bureau voor de Statistiek in Suriname (2012). Censusstatistieken 2012. Recuperado de http://www. statistics-suriname.org/index.php/statistieken/ downloads/category/30-censusstatistieken-2012\#

Gellner, E. (1988). Naciones y Nacionalismo. Madrid: Alianza.

Giménez, G. (2007). Cultura política e Identidad. En Giménez, G. Estudios sobre la cultura y las identidades sociales (pp. 195-214). México: Conaculta-Iteso.

Gleditsch, N. P. (1998). Armed Conflict and the Environment: A Critique of the Literatura. Journal of peace research, 35 (3), 381-400.
González Gutiérrez, C. (2006). Introducción: de Estados y diásporas. En González Gutiérrez, C. (coord.). Relaciones Estado-Diáspora: aproximaciones desde cuatro continentes (pp. 11-21). México, D. F: Miguel Ángel Porrúa.

Government of Canada (2011). Statistics Canada. 2011 National Household Survey: immigration, place of birth, citizenship, ethnic origin, visible minorities, language and religion. Recuperado de http:// www.statcan.gc.ca/daily-quotidien/130508/ dq130508b-eng.htm

Government of the Republic of Trinidad and Tobago, Ministry of Planning and Sustainable Development, Central Statistical Office, 2012). Trinindad and Tobajo 2011 Population and Housing Census. Demographic Report. Recuperado de https:// guardian.co.tt/sites/default/files/story/2011_DemographicReport.pdf

Grupo de Trabajo sobre India, Comité de Asuntos Asiáticos, CARI (2014). Boletín 1 (diciembre). Recuperado de http://www.cari.org.ar/pdf/boletin_indial.pdf

Grupo de Trabajo sobre India, Comité de Asuntos Asiáticos, CARI (2015a). Boletín 2 (febrero). Recuperado de http://www.cari.org.ar/pdf/boletin_india2.pdf

Grupo de Trabajo sobre India, Comité de Asuntos Asiáticos, CARI (2015b). Boletín 3 (abril) (en edición).

Gupta, R. K. (s. f.). Non-State actors in international relations: a case of Indian diaspora in Africa. Recuperado de http://www.iriis.in/pdf/2030452091. pdf

Guyana Bureau of Statistics (2002). Census 2002. Chapter 11: Population Composition. Recuperado de http://www.statisticsguyana.gov.gy/pubs/Chapter2_Population_Composition.pdf 
Hall, I. (2012). India’s New Public Diplomacy. Soft Power and the Limits of Government Action. Asian Survey, 52 (6), 1089-1110.

Hercog, M. y Siegel, M. (2010). Engaging the diaspora in India. UNU-MERIT Working Paper Series. The Netherlands: Maastrich Graduate School of Governance, United Nations University.

Hollifield, J. F. (1992). Migration and International Relations: Cooperation and Control in the European Community. International Migration Review, 26 (2), 568-595.

Hollifield, J. F. (2008). The Politics of International Migration: How Can We "Bring the State Back In”? En Bretel, C. B. y Hollifield, J. F. (eds.). Migration Theories: Talking across Disciplines (pp. 183-237). New York: Routledge.

Hollifield, J. F. (2012). Why do states risk migration? Paper prepared for IPSA's 22nd World Congress of Political Science, Reshaping Power, Shifting Boundaries, July 8-12, 2012, Madrid, Spain.

Homer-Dixon, T. (1999). Environment, Scarcity and Violence. Princeton, NJ: Princeton University Press.

Hymans, J. E. (2009). India's Soft Power and Vulnerability. India Review, 8 (3), 234-265.

Inderfurth, K. F. (2012). U.S India Insight. Washington: Center for Strategic y International Studies.

INEGI (2010). Informativo Oportuno. Censo de Población y Vivienda 2010. Recuperado de http:// www.inegi.gob.mx/inegi/contenidos/espanol/ prensa/contenidos/Articulos/sociodemograficas/ nacidosenotropais.pdf

Janardhanam, V. (2013). Political participation of the Indian Diaspora in the USA. Journal of International and Global Studies, 5 (1), 16-33. Recuperado de http://www.google.com.ar/url?url=http:// www.lindenwood.edu/jigs/docs/volume5Issue1/ essays $/ 16-33$.pdf\&rct=j\&frm $=1 \& q=\&$ esrc $=s \& s$ $\mathrm{a}=$ U\&ei=dcauvKTFBsS2ogT3gin 4Bg\&ved=0cC
MQFjAA\&sig2=YmYqVeMbumBouvQP5f0RiQ\& usg=AFQjcNHxlwzq6Oc8wxptz7AJMiTonKvAqg Jayaram, N. (1998). The Study of indian Diaspora: a multidisciplinary agenda. Bangalore University. Recuperado de http://www.uohyd.ernet.in/ NJWORD 1.htm

Kauffman, T. (2015). The Agendas of Tibetan Refugees. Survival Strategies of a Government-in-Exile in a World of Transnational Organizations. Series: Forced Migration. New York-Oxford: BerghahnUniversity of Oxford.

Kapur, D. (2010). Diaspora, Development, and Democracy: The Domestic Impact of International Migration from India. Oxford: Oxfor University Press.

Khadria, B. (2006). India: skilled migration to developed countries, labour migration to the Gulf. Migración y Desarrollo, 4-37. Recuperado de http://meme.phpwebhosting.com/ - migracion/ modules/ve7/2.pdf

Khanna, P. (2005). Bollystan: India’s Diasporic Diplomacy. London: Foreign Policy Centre. Recuperado de]http://www.paragkhanna.com/pdf/Parag\%20 Khanna_Bollystan.pdf

Kymlicka, W. (1996). Ciudadanía Multicultural. Barcelona: Paidós.

Leclerc, E. (2004). L'invention d'une diaspora indienne: enjeux politiques et sociaux. Ponencia presentada en "Espaces et sociétés aujourd'hui (la géographie sociale dans l'espace et dans l'action)", Rennes, UMR 6590, Université de Rennes (21-22 Oct 2004). Recuperado de http://eso.cnrs.fr/IMG/ pdf/le.pdf

Leclerc, E. (2008). India recentred: The role of Indian diáspora in the globalisation process. Working Paper 2. IMDs Working Paper Series. International Migration and Diaspora Studies Project. Zakir Husain Centre for Educational Studies. School 
of Social Sciences. Jawaharlal Nehru University. New Delhi, India.

Leclerc, E. (ed.) (2011). International and Transnational Political Actors. Case Studies fron the Indian Diaspora. New Delhi: Manohar Publishers \& Distributors - Centre de Sciences Humaines.

León López, L. E. (2013). La población inmigrante en Puerto Rico. Trabajo presentado en la Conferencia de Estudios poblaciones, Universidad de Puerto Rico en Canvey. Recuperado de http:// www.estadisticas.gobierno.pr/iepr/LinkClick.as px?fileticket=3OJ8yIPDQEU\%3D\&tabid $=104$

Leonard, K. (1989). California’s Punjabi Mexican Americans. The World \& I, 4 (5), pp. 612-623.

Lizcano Fernández, F. (2005). Composición étnica de las tres áreas culturales del continente americano al comienzo del siglo xxL. Convergencia, 38, 185232. Recuperado de http://convergencia.uaemex. $\mathrm{mx} / \mathrm{rev} 38 / 38 \mathrm{pdf} /$ LIZCANO.pdf

López Córdoba, E. y Olmedo, A. (2007) La migración internacional, las remesas y el desarrollo: una visión general. INTAL, Integración y Comercio, $27,1-20$.

Mahalingam, M. (2013). India's Diaspora Policy and Foreign Policy: An Overview. Publications GRFDT. Recuperado de http://www.grfdt.com/PublicationDetails.aspx?Type $=$ Articles $\&$ TabId $=30$ Mahapatra, D. (2015). NRIs can now vote in Indian elections without coming here. The Times of India. Recuperado de http://timesofindia.indiatimes.com/india/NRIs-can-now-vote-in-Indian-elections-without-coming-here/articleshow/45862058.cms

Mansingh, L. y Mansingh, A. (1976). Indian Heritage in Jamaica. Jamaica Journal, 10 (2-4), 10-19.

Matthews, M. (1993). The Passport Society: Controlling Movements in Russia and the USSR. Boulder: Westview Press.
Ministerio de Economía, Digestyc (2009). Vl Censo de Población y V de Vivienda 2007. Tomo 1. Características Generales de la población. San Salvador: Ministerio de Economía. Recuperado de http://www.digestyc.gob.sv/index.php/temas/des/poblacion-y-estadisticas-demograficas/ censo-de-poblacion-y-vivienda/publicacionescensos.html

Ministry of External Affairs/MEA (2002). Report of the High Level Committee on Indian Diaspora. New Delhi: Indian Council of World Affairs.

Ministry of Overseas Indian Affairs (s.f.). Emigration Services. Bilateral Agreements. Recuperado de http://moia.gov.in/services.aspx? 1 D $1=81$ \&id= m4\&idp $=81 \&$ mainid $=73$

Ministry of Oveseas Indian Affairs (s. f.). Recuperado de http://moia.gov.in/

Miller, M. J. (1998). International Migration and Global Security. En Poku, N. y Graham, D. T. (eds.). Redefining Security: Population Movements and National Security (pp. 15-27). Westport, ст: Praeger.

Mitchell, M. I. (2012). The Perils of Population Movements in International relations: new directions for rethinking the migration-conflict nexus. Trabajo presentado en el Canadian Political Science Association Annual Meeting, Edmonton, AB.

Mohammad-Arif, A. (2000). The Lobbying Game of the Indian and Pakistani Diasporas in the U.S. Working Paper for Le Dossier. Paris: Centre International Studies and research. Recuperado de http://www.ceri-sciences-po.org

Motwani, J., Gosine, M. y Barot-Motwani, J. (1993). Global Indian Diaspora Yesterday, Today and Tomorrow. New Delhi: Gopio.

Migration Policy Institute/MPI (2014). RAD Diaspora Profile. The Indian Diaspora in the United States. Recuperado de file:///C:/Documents $\% 20$ and $\% 20$ Settings/Administrador/Mis\%20do- 
cumentos/Downloads/RAD-IndiaII-FINAL $\% 20$ (1).pdf

Murti, B. (2013). Indias Use of Digital Media to Engage with Diaspora as Part of its Public Diplomacy Outreach a case study analysis of the website of Overseas Indian Facilitation Centre [Tesis de Maestría]. Washington: The Faculty of the Public Communication Program School of Communication American University Washington, D.C.

Murti, B. y Zaharna, R. S. (2014). India’s Digital Diaspora Diplomacy: Operationalizing Collaborative Public Diplomacy Strategies for Social Media. Exchange Journal of Public Diplomacy, 5, 3-29.

Nijhawan, S. (2003). Why has the Indian Diaspora been shunned by successive Indian governments? [Thesis for MA]. London: International Studies and Diplomacy. School of Oriental and African Studies London, Faculty of Law and Social Sciences, Centre of South Asian Studies.

Noya, J. (2006). Una diplomacia pública para España. DT 11. Madrid: Real Instituto Elcano. Recuperado de http://www.realinstitutoelcano.org/ documentos/247/247_Javier_Noya_Diplomacia_Publica_Espana.pdf

Nye, J. (2004). Soft Power. New York: Public Affairs.

Oficina Nacional de Estadística e Información, República de Cuba (2012). Censo de Población y Vivienda 2012. Resumen Adelantado. Resultados definitivos de indicadores seleccionadosen Cuba, provincias y municipios. Tabla 4. Población por color de la piel, según provincias y municipios. Recuperado de http://www.one.cu/publicaciones/cepde/cpv2012/20131107resumenadelant ado/Tablas/4.pdf

Ogden, Ch. (2011). India and Nuclear Weapons. En Scott, D. Handbook of India's International relations (pp. 290-300). London and New York: Routledge.
Oonk, G. (ed.) (2007). Global Indian Diasporas: Exploring Trajectories of Migration and Theory. Amsterdam: Amsterdam University Press.

Organización Internacional para las Migraciones (orM) (2001). Honduras. Perfil de la Población Inmigrante. Cifras del Censo 2001. Migrantes Internacionales en Mesoamérica. Recuperado de http://163.178.140.43/descargas/documentos/ boletines/Serie\%20Migrantes\%20Internacionales\%20en\%20Mesoamerica/Vol-05_HONDURAs_ Perfil\%20de\%20la\%20Poblaci\%C3\%B3n\%20 Inmigrante.pdf

Organización Internacional para las Migraciones/orm (2010). Previsiones y Tendencias Mundiales. Recuperado de http://www.newwebsite.iom.int/ jahia/Jahia/about-migration/facts-and-figures/ global-estimates-and - trends/lang/es;jsessionid =B47BEEB99F5E659A245594044A55134B. worker01

Organización Internacional para las Migraciones (OIM) (2011). xI Conferencia Sudamericana sobre Migraciones. Brasilia, República Federativa de Brasil, 19, 20 y 21 de octubre de 2011. Migrantes Extracontinentales en Sudamérica. Informe preliminar. Recuperado de http://csm-osumi.org/Archivos/ ConfCsm/Migrantes\%20Extracontinentales\%20 -\%20Preliminar.pdf

Organización Internacional para las Migraciones (OIM) (2012). Perfil Migratorio de Nicaragua 2012. Managua: oIm. Recuperado de http://costarica. iom.int/public/pdf/Perfil_Migratorio_Nicaragua_2012.pdf

Organización Internacional para las Migraciones (OIM) (2012b). Perfil Migratorio del Ecuador 2011. Quito: OIM. Recuperado de http://publications. iom.int/bookstore/free/Perfil_Migratorio_del_ Ecuador2011.pdf

OASIS, $\mathrm{N}^{\circ} 22 \cdot \mathrm{Julio-Diciembre} 2015 \cdot \mathrm{pp} .23-47$ 
Organización Internacional para las Migraciones (OIM) (2013). Perfil Migratorio de Guatemala 2012. Ciudad de Guatemala: orm. Recuperado de http://costarica.iom.int/public/pdf/Perfil_Migratorio_Guatemala_2012.pdf

Oonk, G. (ed.) (2007). Global Indian Diasporas: Exploring Trajectories of Migration and Theory. Amsterdam: Amsterdam University Press.

Palakshappa, T. C. (1980). Indians immigrants in Waikato. En Tiwari, K. (ed.). Indians in New Zealand: studies of a sub-culture (pp. 198-220). Wellington: Price Milburn.

Postelnicu, A. (2010). Diásporas y desarrollo: un estudio sobre el rol de los migrantes en su país de origen a través de la revisión de la literatura. Relaciones Internacionales, 14, 13-37. Recuperado de file:///C:/Documents\%20and $\% 20$ Settings/Administrador/Mis\%20documentos/ Downloads/213-805-3-PB.pdf

Purushothaman, U. (2010). Shifting Perceptions of Power: Soft Power and India's Foreign Policy. Journal of Peace Studies, 17, 2.3. Recuperado de http:// www.icpsnet.org/adm/pdf/1291714915.pdf

Qureshi, M. H. K. (1994). The South Asian Community. Polyphony. Recuperado de http://collections.ic.gc. $\mathrm{ca} / \mathrm{magic} / \mathrm{mt} 65 . \mathrm{html}$

Rajagopal, K. (2015). NRIs can vote from abroad: govt. tells sc. The Hindu. Recuperado de http:// www.thehindu.com/news/national/nris-canvote-through-postal-ballots/article6780906. ece?ref=relatedNews

Reuveny, R. (2007). Climate Change-Induced Migration and Violent Conflict. Political Geography, 26, 656-673.

Rodríguez de la Vega, L. (2006). Las diásporas en las relaciones internacionales. La inmigración india en Argentina; primera y segunda generación [Te- sis doctoral]. El Salvador: Facultad de Ciencias Sociales, Universidad del Salvador.

Rodríguez de la Vega, L. (2014). Cultura, poder y actores no estatales: dinámicas de política doméstica e internacional en la diáspora india. Ponencia presentada en el Seminario Perspectivas de cultura y política en la India (Organizado por el Comité de Asuntos Asiáticos -Grupo de Trabajo sobre India-). Consejo Argentino para las Relaciones Internacionales (CARI), Ciudad Autónoma de Buenos Aires, República Argentina (22 de septiembre) (en edición para publicación).

Rodríguez de la Vega, L. y Meza, M. (2014). La migración india en el Uruguay y su relación con el país de origen y el de residencia: aproximaciones a su estudio. En Onaha, C. y Rodríguez de la Vega, L. (comps.) (2014). Colección ALADAA. Documento 4. Congreso Nacional ALADAA, Sección Argentina, 2014 (pp. 912-932). Buenos Aires: AladaA (libro digital). Recuperado de http://www.aladaa. com.ar/coleccionaladaa/CONGRESO-NACIONALALADAA-ARGENTINA2014.pdf

Rosales Sandoval, M. I. (2009). La perspectiva de la migración internacional en el marco de las relaciones internacionales: las remesas sociales, una nueva reflexión. Ponencia presentada en el Seminario Permanente de Reflexión sobre Migraciones del Instituto Centroamericano de Estudios Sociales y Desarrollo (INCEDES), 10 de septiembre de 2009, Ciudad de Guatemala.

Rudolph, C. (2003). Globalization and Security: Migration and Evolving Conceptions of National Security in Statecraft and Scholarship. Security Studies, 13 (1), 1-32.

Shain, Y. y Barth, A, (2003). Diasporas and International Relations Theory. International Organization, 57 (3), 449-479. Recuperado de http://www.fas. 
nus.edu.sg/migration/newsevents/docs/Diasporas\%20and\%20IR\%20Theory.pdf

Sharma, J. (s.f.). Características de la diáspora india y su relación con el país de origen. Recuperado de http://rimd.reduaz.mx/coleccion_desarrollo_migracion/relaciones-estado1/RelacionesEstado1_ 3 caracteristicas.pdf

Sheffer, G. (ed.) (1986). Modern Diasporas in International Politics. Australia: Croom Helm Ltd.

Singh García, I. (2000). Mexican Hindus. Recuperado de http://samrao.com/aboutasianindianfacts.pdf

Sinha-Kerkhoff, K. y Bal, E. (2003). Eternal Call of the Ganga. Reconnecting with People of Indian Origin in Surinam. Economic and Political Weekly, 38 (38), 4008-4021.

Srivastava, S. y Singh, S. (2014). Public Diplomacy in India: Engaging the Domestic Audience. En CPD. Rissing Soft Powers. India (pp. 1-17). Los Ángeles: usc Center on Public Diplomacy. Recuperado de http://uscpublicdiplomacy.org/sites/uscpublicdiplomacy.org/files/useruploads/u20150/India\%20 soft $\% 20$ power_0.pdf

Tedesco, L. (2008). Remesas, Estado y Desarrollo. Documento de Trabajo 72. Madrid: Fundación para las Relaciones Internacionales y el Diálogo Exterior (FRIDE). Recuperado de http://fride.org/ download/wp_72_remesasyestado_esp_no08.pdf

Therwath, I. (2007). Working for India or against Islam? Islamophobia in Indian American Lobbies. South Asia Multidisciplinary Academic Journal (samaj). Recuperado de http://samaj.revues.org/262

Therwath, I. (2010). Shining Indians: Diaspora and Exemplarity in Bollywood. South Asia Multidisciplinary Academic Journal (SAMAJ). Recuperado de http://samaj.revues.org/3000

Therwath, I. (2012). Cyber-Hindutva: Hindu Nationalism, the diaspora and the web. New Delhi: Centre de Sciences Humaines.
The Economist (2014). Remittances from Indian diaspora on the rise. Recuperado de http://articles. economictimes.indiatimes.com/2014-02-19/ news/47489867_1_indian-diaspora-remittancesoverseas-indians

The Indian Diaspora (s. f.). High Level Committee on Indian Diaspora. Recuperado de http://indiandiaspora.nic.in/contents.htm

The Statistical Institute of Belize (2013). Belize. Population \& Housing Census. Country report, 2010. Recuperado de http://www.sib.org.bz/ Portals/0/docs/publications/census/2010_Census_Report.pdf

Thussu, D. (2014). De-Americanizing Soft Power Discourse? En CPD. Rissing Soft Powers. India (pp. 1-17). Los Ángeles: usc Center on Public Diplomacy. Recuperado de http://uscpublicdiplomacy. org/sites/uscpublicdiplomacy.org/files/useruploads/u20150/India\%20soft\%20power_0.pdf US Census (2010). The Asian Population: 2010. 2010 Census Briefs. Recuperado de http://www.census. gov/prod/cen2010/briefs/c2010br-11.pdf

Varadarajan, L. (2010). The Domestic Abroad: Diasporas in International Relations. Oxford: Oxford University Press.

Vertovec, S. (1995). Indian indentured migration to the Caribbean. En Cohen, R. (ed.). The Cambridge survey of world migration (pp. 57-62). Cambridge: Cambridge University Press.

Vertovec, S. y Cohen, R. (1999). Migration, Diasporas and Transnationalism. Massachusetts: Edward Elgar Publishing Limited.

Weiner, M. (1985). On International Migration and International Relations. Population and Development Review, 11 (3), 441-455.

Wendt, A. (1995). Constructing International Politics. International Security, 20 (1), 71-81. 
Xiang, B. (2005). Relaciones internacionales y migraciones transnacionales: el caso de China. Revista Cidob D'afers Internacionals, 6 8, 133-149. Recuperado de file:///C:/Documents\%20and\%20 Settings/Administrador/Mis\%20documentos/ Downloads/68xiang.pdf

Zeraoui, Z. (2006). Migración y Relaciones Interna- cionales. sedici. Repositorio Institucional de la UNLP. Recuperado de http://sedici.unlp.edu.ar/ handle/10915/9771

Zolberg, A. y Woon, L. (1999). Why Islam is Like Spanish: Cultural Incorporation in Europe and the United States. Politics and Society, 27 (1), 5-38. 